\title{
Dieux et lieux de la Méditerranée antique : des outils numériques pour l'histoire des religions
}

Gods and Places of the Ancient Mediterranean: Digital Tools for the History of Religions

\section{Élodie Guillon et Antoine Laurent}

\section{OpenEdition}

\section{Journals}

Édition électronique

URL : https://journals.openedition.org/revuehn/1451

DOI : 10.4000/revuehn. 1451

ISSN : 2736-2337

Éditeur

Humanistica

Référence électronique

Élodie Guillon et Antoine Laurent, « Dieux et lieux de la Méditerranée antique : des outils numériques pour l'histoire des religions », Humanités numériques [En ligne], 3 | 2021, mis en ligne le 01 mai 2021, consulté le 15 juillet 2021. URL : http://journals.openedition.org/revuehn/1451 ; DOI : https://doi.org/ 10.4000/revuehn. 1451

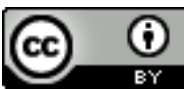

Les contenus de la revue Humanités numériques sont mis à disposition selon les termes de la Licence Creative Commons Attribution 4.0 International. 


\title{
humanités numériques
}

3 | 2021

Humanités numériques spatialisées

SYSTÈMES D'INFORMATION GÉOGRAPHIQUE ET ARCHÉOLOGIE

\section{Dieux et lieux de la Méditerranée antique : des outils numériques pour l'histoire des religions}

\author{
Gods and Places of the Ancient Mediterranean: \\ Digital Tools for the History of Religions
}

\section{Élodie Guillon et Antoine Laurent}

\section{Résumés}

L'article présente plusieurs outils numériques du projet ERC MAP (Mapping Ancient Polytheisms), qui traite d'histoire des religions dans la Méditerranée antique. Ce projet s'est saisi de systèmes de gestion de base de données (SGBD), de webmapping et d'outils de formalisation, afin de pouvoir traiter une documentation conséquente mais disparate, à l'étendue chronogéographique inédite pour le sujet. L'article met l'accent sur les choix et les étapes de la mise en œuvre des outils, qui répondent aux critères des données FAIR. L'idée est de mettre en lumière un exemple concret de projet scientifique pluridisciplinaire, à la croisée de l'histoire et des pratiques numériques.

This article presents several digital tools used in the ERC MAP project (Mapping Ancient Polytheisms), which deals with the history of religions in the ancient Mediterranean. The project uses database management systems (DBMS), webmapping and formalization tools, in order to deal with substantial but heterogeneous documentation, with a chrono-geographical scope unique in the field. The article focuses on the choices and stages of implementation of tools that respect the FAIR data principles. It provides a concrete example highlighting a multidisciplinary scientific project at the crossroads of history and digital practices. 


\section{Entrées d'index}

MOTS-CLÉS : humanités numériques spatialisées, histoire, base de données, histoire antique, sciences des religions, système d'information géographique, cartographie en ligne, données ouvertes

KEYWORDS: spatial digital humanities, history, ancient history, database, religious studies, geographic information system, webmapping, open data

Le projet de recherche ERC Advanced Grant intitulé Mapping Ancient Polytheisms. Cult Epithets as an Interface between Religious Systems and Human Agency $\left(M A P^{1}\right)$, qui a débuté en 2017 pour cinq années à l'université Toulouse-Jean-Jaurès, porte sur les noms divins et les processus de dénomination du divin par les hommes dans les mondes grecs et les mondes sémitiques de l'Ouest (araméen, hébreu, phénicien, punique...). Il s'intéresse en particulier à la partie du nom de la divinité traditionnellement identifiée comme l'épithète ou l'épithète cultuelle (épiclèse) (Bonnet et al. 2018), autrement dit la partie du nom qui qualifie la divinité en mentionnant un champ ou un domaine d'action, une qualité du dieu, un lieu auquel il est attaché. Prenons pour exemple le cas de Zeus. La documentation nous livre de nombreuses épithètes qui lui sont associées : Zeus sôter (sauveur), Zeus ouranios (céleste), Zeus keraunios (de la foudre), Zeus xenios (hospitalier), Zeus meilichios (doux), etc. Ces épithètes accompagnent les noms des dieux dans plus de la moitié des cas jusque-là recensés (et il ne s'agit que d'une première estimation).

\section{Des dieux et des hommes. Présentation et problématiques du projet MAP}

Le projet MAP repose sur le postulat que les noms des divinités forment un langage, comme une clé d'accès aux modes de représentation et de structuration du divin par les hommes. Certaines qualifications sont partagées, d'autres sont exclusives, certaines sont activées dans des contextes précis, d'autres dans des contextes plus globaux. Le projet ambitionne ainsi de dépasser l'image qui a longtemps prévalu dans l'histoire des religions de panthéons en arbres généalogiques statiques, constitués de dieux-personnes, unis par des liens de parenté et désignés par un label univoque et définitif : Aphrodite, pour donner un seul exemple, y est déesse de l'amour, épouse d'Héphaïstos, dieu du feu, maîtresse d'Arès, dieu de la guerre, etc. Son "équivalent " sémitique, Astarté, est décrite peu ou prou de la même manière. Au contraire, le projet propose une lecture des religions anciennes aussi complexe et fluide que le laisse percevoir la documentation. Le cas de la déesse sémitique Ishtar est particulièrement éloquent ; elle échappe à toute catégorisation fixe et univoque : elle n'est jamais liée de la même manière aux autres divinités : par exemple elle est la fille tantôt du dieu An, tantôt du dieu Sîn ou encore d'Ea ; elle possède des identités multiples (Ishtar de Ninive n'est pas la même qu'Ishtar d'Arbèle), et ses domaines d'action sont également variables (Guillon et Porzia 2018). 
Le projet est ainsi un héritier des travaux de Jean-Pierre Vernant qui affirmait déjà en 1965 que les dieux sont des puissances, et non des personnes (Vernant 1965, 1974). Chaque dieu est donc une sorte de champ magnétique, avec ses polarités et son dynamisme, susceptible d'agencements, de redéfinitions, de modulations en fonction des contextes historiques concernés. Dans ce système, ce sont précisément les qualifications qui expriment ce que sont les dieux, ce qu'ils font, leurs aptitudes, leurs modes d'action ou leurs spécificités en tel lieu ou tel autre, à telle époque ou telle autre, en telle ou telle circonstance.

Cela ne signifie pas pour autant que les théonymes seraient une base fixe à laquelle on attache les épithètes (les qualifications) comme des vêtements à un portemanteau. En effet, après trois ans passés à étudier la documentation grecque comme sémitique, il est évident que le binôme théonyme-épithète ne fonctionne pas toujours et sa systématicité impliquerait de rigidifier et de simplifier les sources. C'est là que réside d'ailleurs l'originalité du projet MAP : envisager la documentation dans sa totalité et proposer la première étude d'ensemble des dénominations divines au sens large. La première étape de ce travail est un renouvellement conceptuel et lexical de la manière d'envisager l'onomastique divine. Plutôt que d'employer à nouveau théonymes, épithètes et épiclèses, on parlera dorénavant d'élément onomastique, afin de désigner sans $a$ priori chaque mot ou ensemble de mots qui vient composer le nom divin. Ce dernier est donc une séquence onomastique, plus ou moins longue, plus ou moins complexe (Bonnet et al. 2018).

L'utilisation des termes d' "élément " et de "séquence onomastique " permet ainsi une enquête sur la structuration des noms et, à travers eux, sur celle des représentations du divin, d'une part ; sur les stratégies humaines, individuelles et collectives qui visent à rendre l'interaction avec les divinités aussi efficace que possible en " convoquant " tel ou tel interlocuteur précis, d'autre part. Ces stratégies supposent par conséquent qu'il existe un ensemble de savoir-faire, de normes, de formes d'appropriation locales, mais également d'expérimentation dans ces processus d'adresse au divin ou de sa désignation.

Un tel renouvellement conceptuel et de tels objectifs scientifiques s'accompagnent nécessairement d'une réflexion sur les outils à mettre en œuvre pour soutenir les recherches de l'équipe. Le projet s'appuie naturellement sur les seuls à même d'envisager la documentation dans son ensemble, les outils des humanités numériques. Le corpus, comme on l'a mentionné plus haut est en effet particulièrement étendu, couvrant les mondes grecs dans leur plus grande extension, ainsi que les mondes sémitiques de l'Ouest et leurs diasporas méditerranéennes. Davantage que géographiques, les limites du corpus sont linguistiques : toutes les inscriptions en grec et en langues sémitiques de l'Ouest sont prises en compte. Une première estimation montre qu'elles sont globalement situées dans une grande Méditerranée (figure 1). En termes chronologiques, l'empan considéré s'étend du début du I ${ }^{\mathrm{er}}$ millénaire avant notre ère, jusqu'au $\mathrm{IV}^{\mathrm{e}}$ siècle de notre ère. Ainsi, cela permet d'englober les premières inscriptions sémitiques, qui voient le jour avec l'émergence des royaumes phéniciens et araméens, puis tous les témoignages grecs et sémitiques des métropoles comme des diasporas - les cités fondées notamment par les Phéniciens et les Grecs jusqu'au Portugal actuel - jusqu'à l'affirmation du christianisme dans l'Empire romain. 


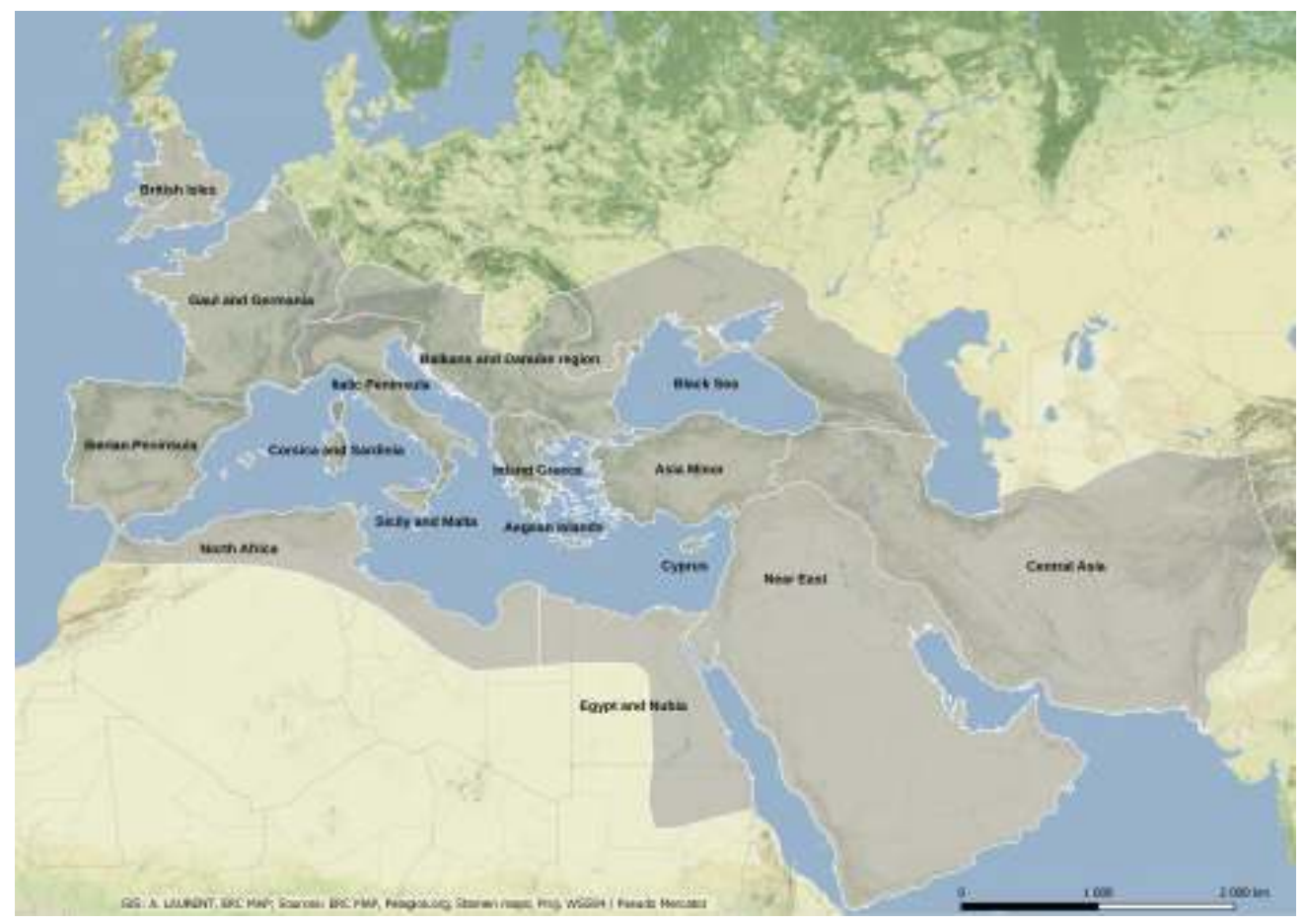

Projet ERC MAP. Licence Creative Commons BY-NC-SA

La nécessité de construire une base de données s'est imposée dès l'écriture du projet, afin de prendre en compte une documentation fournie (quelques milliers, voire dizaines de milliers d'inscriptions épigraphiques, sans compter les sources dites littéraires), mais également disparate et lacunaire. L'ambition du projet MAP est de répertorier exhaustivement l'ensemble des séquences onomastiques divines, au moins dans les sources épigraphiques, et de les rendre accessibles à la communauté scientifique. À partir de cette base de données, l'objectif est également de cartographier les mondes divins, ainsi que l'ancrage du divin dans l'aire chronogéographique définie plus haut. En effet, l'idée n'est pas de fournir un simple répertoire de noms divins, mais bien de se servir des outils numériques pour consulter, croiser, analyser et visualiser les sources du corpus. 


\section{Une Méditerranée remplie de dieux : les problématiques spatiales du projet}

C'est dans ce contexte et ces objectifs qu'entrent en jeu les systèmes d'information géographique (SIG), c'est-à-dire les logiciels qui permettent d'acquérir, d'organiser, de gérer, de traiter et de restituer des données géographiques sous forme de plans et cartes. Le sigle du projet, $M A P$, dit bien en effet l'importance de la notion d'espace, qu'il soit un construit idéel - l'espace des dieux - ou bien l'espace géographique (Brunet, Ferras et Théry 2005). Le projet travaille dans deux directions. La première est de tenter de mieux connaître la structure des panthéons anciens à partir des noms divins, ainsi que leurs évolutions selon les lieux, les époques et les contextes particuliers. La seconde est celle des liens entre les hommes et les divinités, en d'autres termes, la question de l'agency humaine : que nous dit l'invocation d'un élément onomastique dans un lieu plutôt qu'un autre, un territoire plutôt qu'un autre ? Le transfert d'un élément d'un endroit à un autre ? Par ailleurs, un rapide regard sur la documentation montre clairement que les éléments topographiques et toponymiques sont très fréquents dans les séquences, tant dans les mondes grecs que les mondes sémitiques (Parker 2017 ; Smith 2016). Ancrer les dieux dans l'espace humain, l'espace vécu, semble donc un enjeu primordial des hommes qui s'adressent à eux.

Prenons un exemple, celui du dieu phénicien tutélaire de Tyr, Melqart, qu'on retrouve à Tyr, évidemment, mais également en Sardaigne, à Carthage, à Gadès ou à Ibiza. Melqart est le « souverain " de Tyr, le Baal Sour. Ce nom joue sur le mot Sour, à la fois le rocher et "Le " rocher primordial, à savoir la ville de Tyr, originellement fondée sur une île rocheuse. Le retrouver comme "préposé au rocher " en contexte diasporique, c'està-dire dans différentes colonies phéniciennes de Méditerranée, n'a rien d'étonnant. En revanche, sa présence pose la question des relations entre ces colonies et la métropole. Deux auteurs anciens, Diodore et Justin évoquent les liens particuliers entre Gadir (Cadix), Carthage et Tyr (Bonnet 2015). Mais qu'en est-il d'Ibiza, qui est une fondation phénicienne dont la cité mère n'est pas Tyr, mais très probablement une cité du sud de la péninsule Ibérique ? Et quels sont encore les contextes d'invocation du dieu en Sardaigne ? La distance, spatiale ou temporelle, joue très certainement un rôle dans la réactivation de la qualification du dieu en lien avec Tyr, qu'il reste à définir précisément.

Les questions spatiales sont donc diverses. Elles se posent dans des aspects linguistiques - comment exprime-t-on l'attachement d'un dieu à un lieu ? (Smith 2016, $2^{2}$ ) - mais aussi dans des aspects géographiques. Les sources portant les séquences onomastiques peuvent être géolocalisées et montrer à des échelles macro et micro des répartitions, des diffusions ou des transferts de noms divins. Par ailleurs, l'analyse des contextes d'emploi de telles séquences onomastiques est révélatrice de différents enjeux : des trajectoires politiques de divers sites et cités, des oppositions entre différents sanctuaires (antériorité, légitimité, avantages économiques), des déplacements humains, des processus de colonisation, d'expansion ou de territorialisation, la recherche de légitimation de la part de communautés, etc. Ce n'est pas l'analyse de 
chaque contexte qui est innovante (elle est souvent déjà avancée par les spécialistes de la question), mais bien leur mise en série, qui pourrait révéler des typologies, des tendances, peut-être aussi des grammaires. Dénomination divine et questions spatiales sont en effet trop rarement croisées ou de manière isolée et ponctuelle. L'aire d'enquête est également plurielle, et la question de l'interaction du divin avec l'environnement est fondamentale. Quelles sont les séquences onomastiques liées à la mer, ou au contraire aux territoires intérieurs, aux montagnes, aux îles?

Prenons un autre exemple, sur l'île d'Ibiza. Entre 2015 et 2017, le projet PPI a recensé l'ensemble des sites archéologiques découverts sur l'île et sa voisine Formentera afin d'étudier les différentes phases d'occupation du sol entre l'arrivée des Phéniciens au viI ${ }^{\mathrm{e}}$ siècle avant notre ère et l'insertion progressive de l'île dans le monde romain à partir du $\mathrm{II}^{\mathrm{e}}$ siècle avant notre ère ${ }^{3}$. Les implantations ont été soumises à une étude typologique, fondée sur les données archéologiques et une analyse statistique multivariée, puis leur répartition a fait l'objet d'une analyse spatiale, grâce à l'utilisation d'un SIG. C'est une véritable colonisation agricole que suggèrent les résultats de ces analyses, organisée et planifiée par un établissement situé en position centrale de l'île, la cité d'Ibiza (Guillon $2021^{4}$ ). Cette colonisation est particulièrement remarquable dans le nord de l'île, autour de la Cala de Sant Vincent, en particulier aux $\operatorname{III}^{\mathrm{e}}$ et II $^{\mathrm{e}}$ siècles (Gómez Bellard, Díes Cusí et Marí i Costa 2011). Or, à la même période, un sanctuaire s'établit et connaît un fonctionnement particulièrement intense, qui semble aller de pair avec la densification significative de l'implantation humaine.

Ce dernier est dédié à la déesse Tanit, qualifiée, dans une inscription du sanctuaire, de Gad. L'étude de cet élément onomastique nous emmène au Proche-Orient, où on le trouve associé à des divinités phéniciennes dont la sphère d'activité comprend la protection des groupes humains et donc des villes ${ }^{5}$. D'autres indices iconographiques, comme les représentations des divinités Gad, semblables au Proche-Orient et à Ibiza, paraissent confirmer que c'est bien Tanit-Gad qui est implantée dans ce sanctuaire (Marín Ceballos et al. 2010). Ainsi, la mise en relation de l'analyse des éléments onomastiques, ici Gad, mis en série, combinée à l'analyse spatiale d'un environnement précis, ici Ibiza, aboutit à une meilleure compréhension des relations entre la divinité Tanit-Gad et la cité dans laquelle est implanté le sanctuaire. L'hypothèse est donc faite que, au moins à partir de la fin du $\mathrm{III}^{\mathrm{e}}-\mathrm{II}^{\mathrm{e}}$ siècle avant notre ère, Tanit (Gad) est choisie comme divinité poliade, protectrice d'Ibiza, de ses habitants plus généralement et du territoire qu'ils occupent. Il s'agirait alors d'un rôle équivalent à celui qu'elle exerçait à Carthage. L'élément Gad devra donc être étudié dans d'autres contextes en Méditerranée occidentale, afin d'entrer dans le détail des liens entre les divinités Gad et leurs territoires d'implantation phéniciens et puniques.

Cet exemple illustre une des différentes approches spatiales des données. D’autres visent à identifier des répartitions, des réseaux, des itinéraires, dans une première étape. La suivante est d'inclure la dimension temporelle, en étudiant l'évolution de ces configurations au cours du temps, selon les contextes. L'enjeu est alors double. D'une part, chaque aire géoculturelle connaît ses propres changements (conflits, reconfigurations politiques, processus de colonisation...), et d'autre part, chacune 
peut également faire l'objet de comparaisons : le projet est intrinsèquement comparatiste et il sera intéressant de voir comment réagissent les processus de dénomination divine à certains changements, par exemple la conquête d'Alexandre et la période hellénistique, la conquête romaine et la constitution de l'Empire, etc.

\section{Qui sont ces dieux? Panorama du corpus et de la base de données du projet}

Si l'on a déjà dit un mot des sources et du type de données que le projet recense, il est toutefois nécessaire de redire un mot du corpus et de présenter brièvement la base de données avant d'en venir plus concrètement aux outils de cartographie et d'analyse spatiale mis en œuvre dans le projet.

L'étape initiale de collecte et d'analyse des besoins de l'équipe scientifique a été cruciale dans le choix du traitement de l'information (Laurent et al. 2019). Tout d'abord, le corpus est hétéroclite sur plusieurs de ses aspects. Le support physique des sources est très variable allant d'une inscription sur un fronton de temple à un ostracon, en passant par une ancre de bateau. Ensuite, la sélection de la donnée extraite des textes se concentre sur la séquence onomastique (Bonnet et al. 2018) et sur les paradonnées du contexte historique. La caractérisation du matériau épigraphique, porteur de la donnée de base, s'effectue sur des analyses quantitative et qualitative multi-échelle et non sur des éléments de linguistique. Cette étape de plusieurs mois a permis de s'orienter vers une base de données de type relationnelle en SQL plutôt que vers des outils en XML.

La conception du système d'information est fondée sur la méthode MERISE $^{6}$, développée dans les années 1970. Elle possède trois niveaux pour l'élaboration de l'infrastructure (conceptuel, logique et physique) que nous avons suivis pour la création de la base MAP. L'élaboration du dictionnaire de données comprenant l'ensemble des champs d'intérêt et/ou porteurs de requête a permis de dégager une structure hiérarchique à trois niveaux emboîtés. En archéologie préventive, un site de fouilles contient des sondages qui, eux-mêmes, contiennent des vestiges et ces derniers peuvent être observés dans plusieurs sondages tel un mur qui se prolonge. Pour MAP, une source (support physique de l'information) contient des attestations (passage d'une source contenant les noms divins) qui sont composées d'un ou plusieurs éléments (mot ou ensemble de mots faisant sens composant le nom divin). La description de l'information collectée dans les différentes entités privilégie l'utilisation d'ontologies et de thésaurus déjà existants dans chaque domaine du projet, à l'image de l'utilisation des PACTOLS ${ }^{7}$ pour la description des supports et des matériaux de la source.

Plusieurs entités se forment autour des trois principales comme la datation, la localisation, les agents ou encore la bibliographie. De nombreuses tables, contenant des listes de valeurs bilingues et décrivant le corpus, gravitent autour de celles-ci. Les associations entre les entités interviennent à plusieurs niveaux. Nous retrouvons ainsi la donnée spatiale pour diverses raisons : une source possède un lieu d'origine, un lieu 
de découverte, ou les deux ; une attestation mentionne un endroit ; un agent peut mentionner, sur certaines inscriptions, sa propre origine ; un élément fait référence à une autre localisation.

Afin d'évaluer la précision des données parfois lacunaires, l'équipe scientifique a développé deux indicateurs de précision pour la datation et pour la localisation de la source ainsi qu'un indicateur de qualité de lecture de l'attestation. Cette réflexion collective aboutit à l'élaboration du Modèle conceptuel de données (MCD) (figure 2).

FIGURE 2. MODĖLE CONCEPTUEL DE DONNÉES DU PROJET MAP

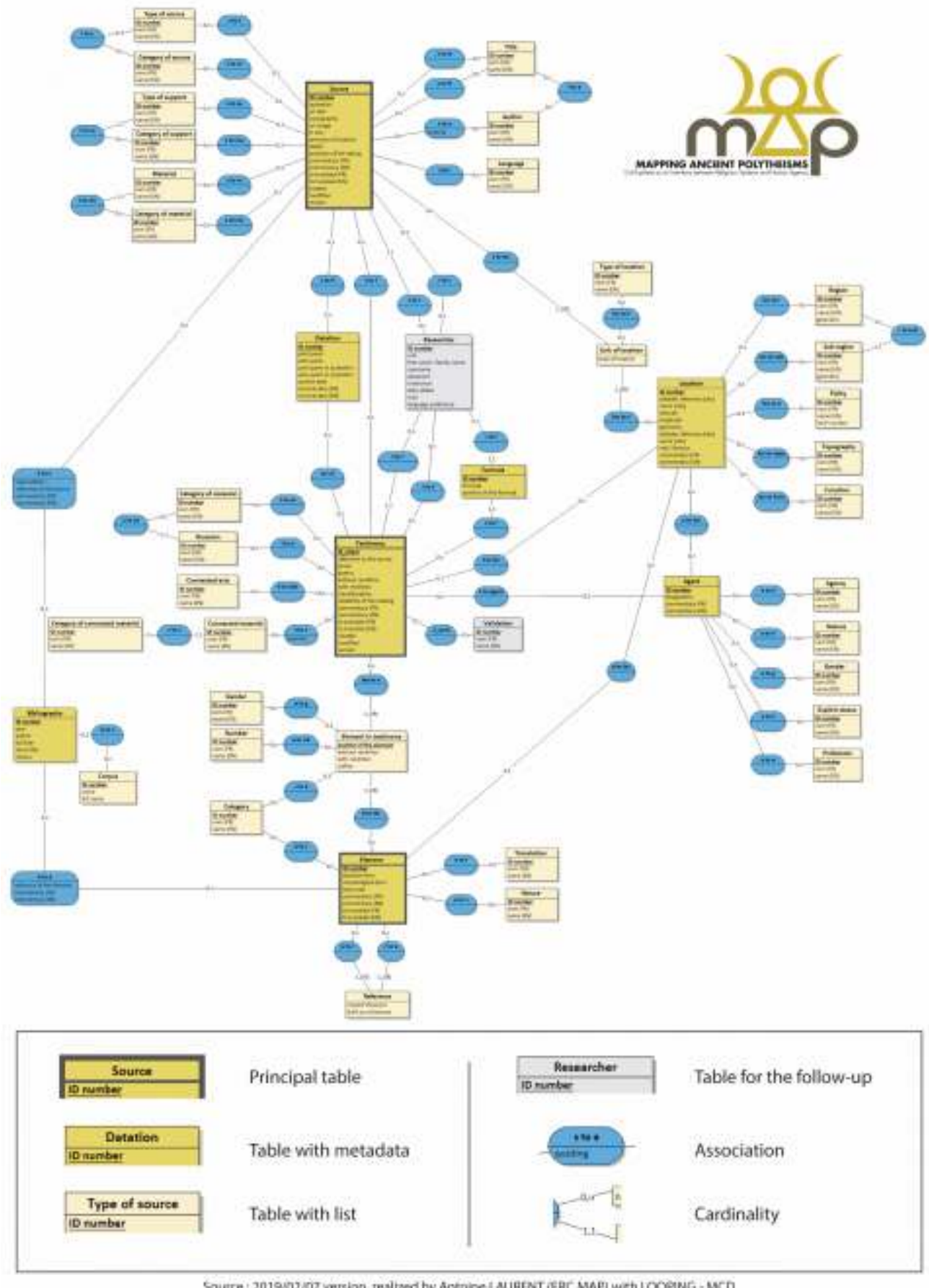

Projet ERC MAP. Licence Creative Commons BY-NC-SA

La base a donc été conçue à partir des sources, pour prendre en compte leurs spécificités et leur diversité. Elle doit, à terme, contenir l'ensemble exhaustif des séquences onomastiques présentes sur des supports épigraphiques, mais elle peut également s'adapter à l'enregistre- 
ment de séquences provenant d'autres sources, telle la Bible ou les sources "littéraires " classiques. C'est donc pour répondre aux problématiques spécifiques du projet que les outils ont été choisis, avec un objectif : le FAIR data.

\section{Les dieux numériques : choix des outils}

Le passage aux niveaux logique et physique de la méthode MERISE s'est effectué à partir des standards et des formats les plus interopérables possible. Dans cette même perspective et dans le respect de l'open access, nous avons fait le choix d'utiliser majoritairement des solutions open source ou de nous appuyer sur des licences offertes par la très grande infrastructure de recherche (TGIR) Huma-Num ${ }^{8}$ qui permet une mise à disposition des données et des interfaces sur le long terme. Toutes les informations sur nos outils numériques sont décrites dans le plan de gestion des données (PGD) mis à jour annuellement, disponible sur la plateforme GitHub ${ }^{9}$ qui offre un accès au code source des interfaces. La structure logique de notre base de données est réalisée avec le logiciel Looping-MCD ${ }^{10}$ développé sur le pôle universitaire toulousain et permettant la génération du code SQL. La technologie utilisée pour la base de données est PostgreSQL ${ }^{11}$ avec l'extension PostGIS pour le traitement des données spatiales.

Le référentiel commun du projet $M A P$ est conçu pour être multi-utilisateur et accessible sur Internet. Nous profitons, là encore, des bienfaits de la TGIR Huma-Num pour l'hébergement et la mise en ligne du système d'information et des outils s'y connectant. En effet, la stratégie est de dissocier les interfaces de la base données, car cela permet de conserver le travail collectif sans le rendre dépendant d'une technologie. Tous les outils accèdent au même silo d'information et des passerelles existent entre eux. L'interface de saisie en ligne (figure 3), réalisée avec Symfony ${ }^{12}$, est la seule qui puisse créer, modifier ou supprimer de l'information. La pérennisation d'une base de données d'un projet est liée à l'activité et à la satisfaction qu'elle procure. À cet effet, le système d'information est conçu pour s'élargir à d'autres projets similaires et proposera des outils de requêtes complets lors de son ouverture au grand public.

FIGURE 3. INTERFACE DE SAISIE EN LIGNE DES SOURCES

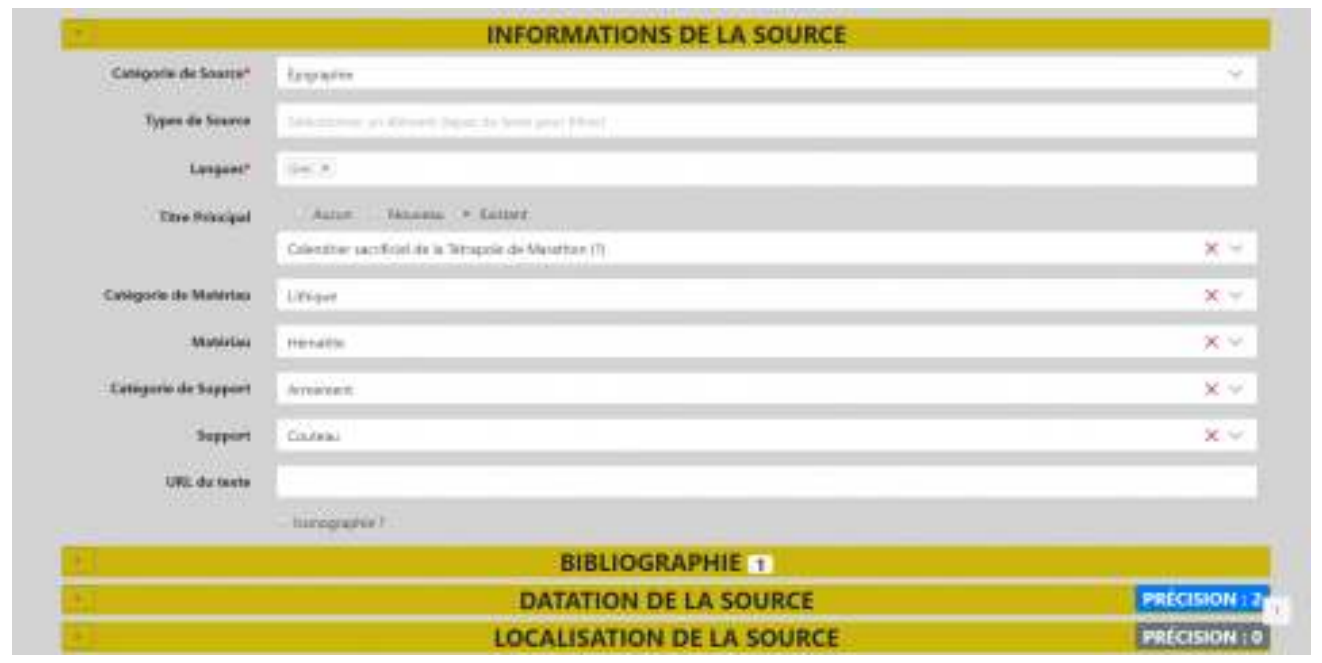

Projet ERC MAP. Licence Creative Commons BY-NC-SA 


\section{Cartographier le divin : webmapping, réseaux et visualisation}

Les référentiels cartographiques sur Internet se sont multipliés depuis le lancement de Google Maps, le plus grand service de cartographie en ligne. Depuis 2004, la visualisation et l'interrogation de la donnée spatiale sont rendues accessibles au plus grand nombre. Le projet MAP s'est approprié cet outil et l'a intégré dans sa réflexion initiale pour l'analyse et la diffusion de son travail de recherche. L'interface est développée avec Géo Générateur ${ }^{13}$ de Business Geographic. Cette solution propriétaire a l'avantage de générer en quelques semaines les cartes en ligne. Un autre aspect de ce choix est la maintenance offerte par la TGIR HumaNum, bien au-delà des missions contractuelles et du temps du projet. L'interface cartographique donne accès à la donnée de la base à l'utilisateur sans possibilité de l'éditer (figure 4). Sur celle-ci, le chercheur ou l'amateur éclairé peut utiliser les outils de webmapping de deux manières. Une solution est la création d'une recherche via l'interface de requête qui génère une sélection sur la carte. L'autre possibilité est d'explorer la mappemonde et de naviguer selon ses problématiques, à l'aide des outils de requête et d'analyse thématique accessibles sur l'interface. Celle-ci permet en effet des affichages par région, mais propose aussi un outil de recherche portant sur les sources, les attestations, les éléments, les agents et même sur les numéros d'identifiants de la base. Ainsi, les utilisateurs peuvent travailler sur des sources matérielles, des agents du culte ou encore des noms de dieux en particulier (figure 5).

Pour aller plus loin, l'équipe scientifique peut se connecter à la base de données grâce à des logiciels d'administration du système d'information ou à des logiciels SIG. Ces interfaces permettent d'utiliser des outils avancés d'analyse spatiale.

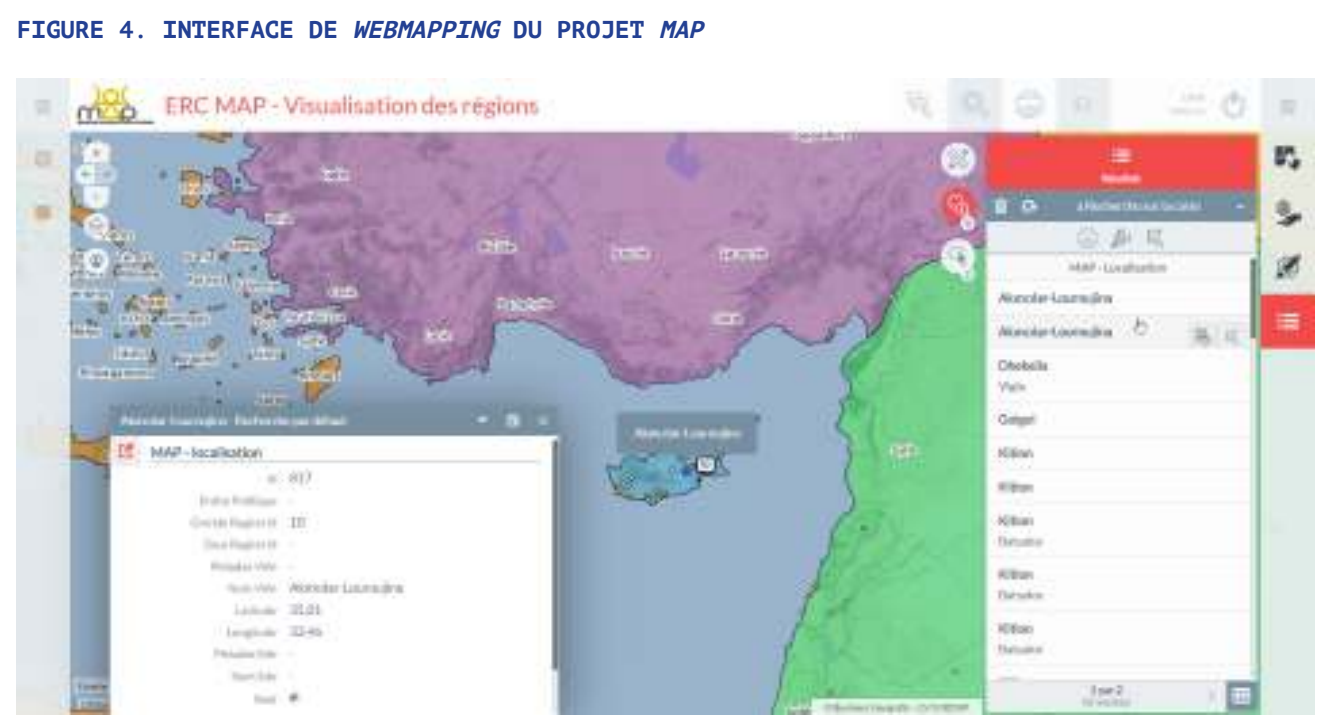

Projet ERC MAP. Licence Creative Commons BY-NC-SA 


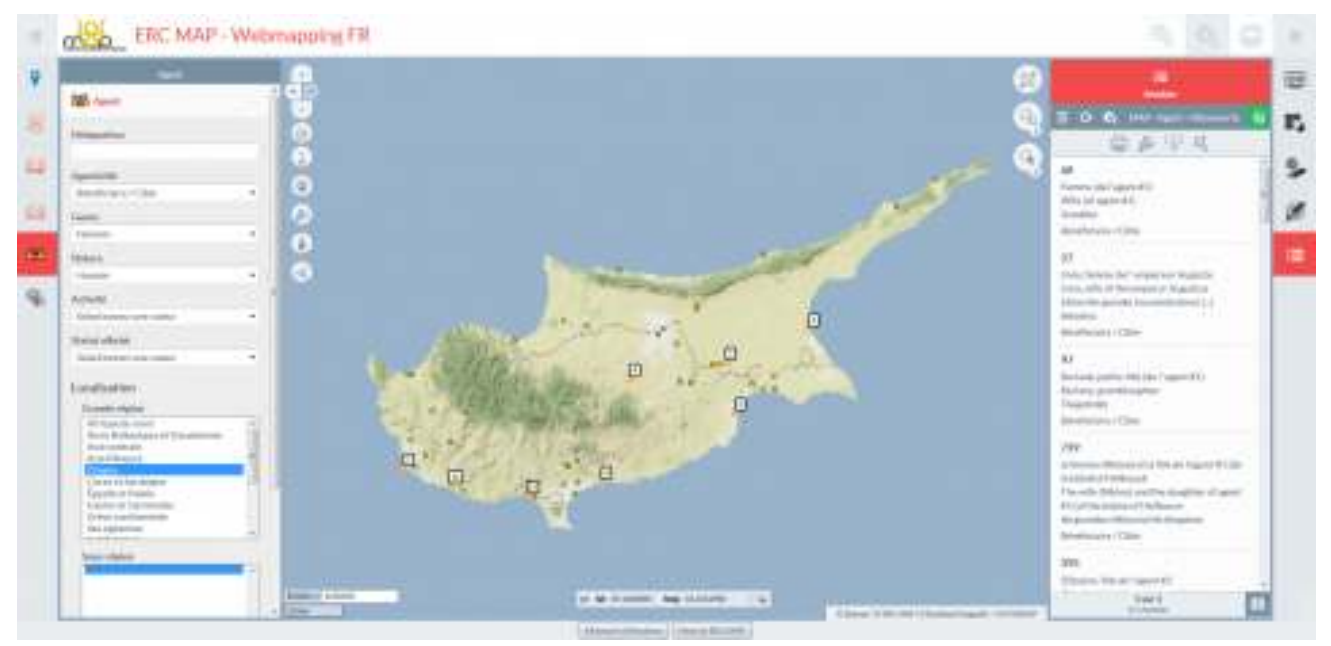

À gauche : formulaire de requête (concernant les femmes bénéficiaires dans des dédicaces à Chypre). À droite : liste des résultats.

Projet ERC MAP. Licence Creative Commons BY-NC-SA

La cartographie du divin peut en outre se dissocier du monde physique et être perçue au travers des outils de Social Network Analysis (SNA). Le projet MAP propose deux sources d'information pour générer et visualiser les graphes. La première est directement liée à la structure de la base de données. Par exemple, l'arborescence hiérarchique permet d'obtenir assez facilement, grâce à des requêtes SQL, un graphe de cooccurrence des éléments contenus dans des sources partageant des critères communs. La seconde solution s'appuie sur une création du projet $M A P$ : les formules onomastiques. Il s'agit d'une formalisation de la séquence onomastique, proposée par les chercheurs du projet, qui tente de saisir la qualité des liens entre les éléments de la séquence. Quatre opérateurs formalisés ont été identifiés, à partir de la documentation :

- La coordination (+) quand deux éléments sont coordonnés. Ex. : Zeus et Héra qui sont à lécoute.

- La juxtaposition (/), quand deux éléments se trouvent dans une séquence sans autre indication. Ex. : Isis Aphrodite.

- La qualification (\#) quand un élément exprime la qualité d'un autre. Ex. : Tanit la puissante ou Aphrodite de la bonne navigation.

- L'équivalence (=) quand un élément est annoncé comme l'équivalent d'un autre. Ex. : Ammôn qui est aussi Chnoubis.

Par ailleurs, les chercheurs utilisent également les crochets [] pour signaler les syntagmes ainsi que les parenthèses () lorsqu'un élément se rapporte à plusieurs autres (dans l'exemple "Zeus et Héra qui sont à lécoute ", "qui sont à l'écoute " se rapporte aux deux dieux). Des graphes peuvent donc être générés à partir des formules et mettent l'accent non plus sur la présence conjointe d'éléments, mais sur la force des liens qui les relient. Ils suscitent ainsi des interrogations et des hypothèses différentes, en cours, sur la formation des panthéons (figure 6). 


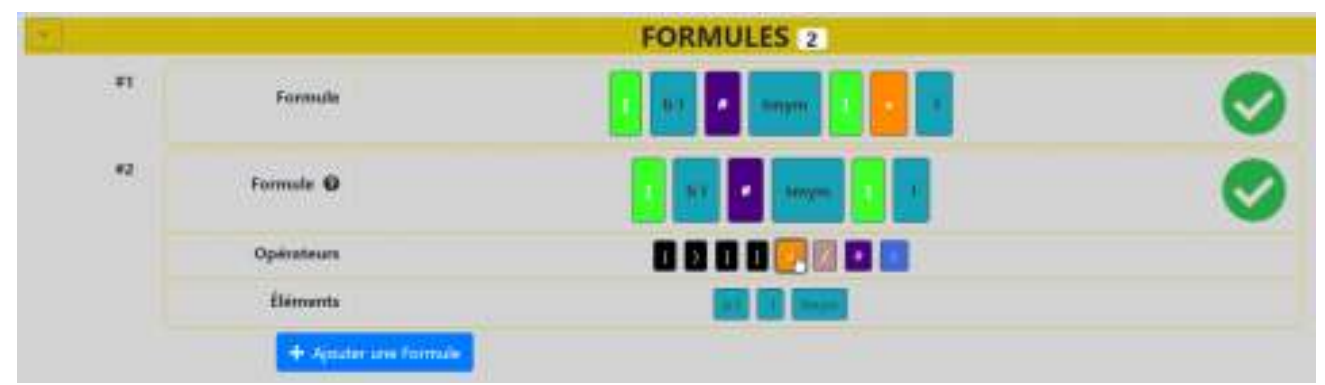

Projet ERC MAP. Licence Creative Commons BY-NC-SA

Un outil de modélisation et de visualisation des graphes a été créé par S. Köhler afin d'amener les spécialistes directement à la phase d'analyse (figure 7). À partir des formules onomastiques exportées depuis l'interface de requête, le programme permet de générer des graphes de deux manières. Il se base sur la cooccurrence ou sur la structure des formules et ses opérateurs. Cette dernière méthode pondère le poids des liens entre les éléments à l'aide de la proximité exprimée par les parenthèses ou les crochets. Ainsi deux éléments dans le même crochet auront des liens plus forts qu'avec les autres éléments de la chaîne onomastique (Köhler 2019). Cette application, nommée Map Graphe, est développée avec le langage Python et sera transposée à l'avenir sur l'interface en ligne.

FIGURE 7. GÉNÉRATION DE GRAPHES À PARTIR DE L’OUTIL MAP GRAPHE

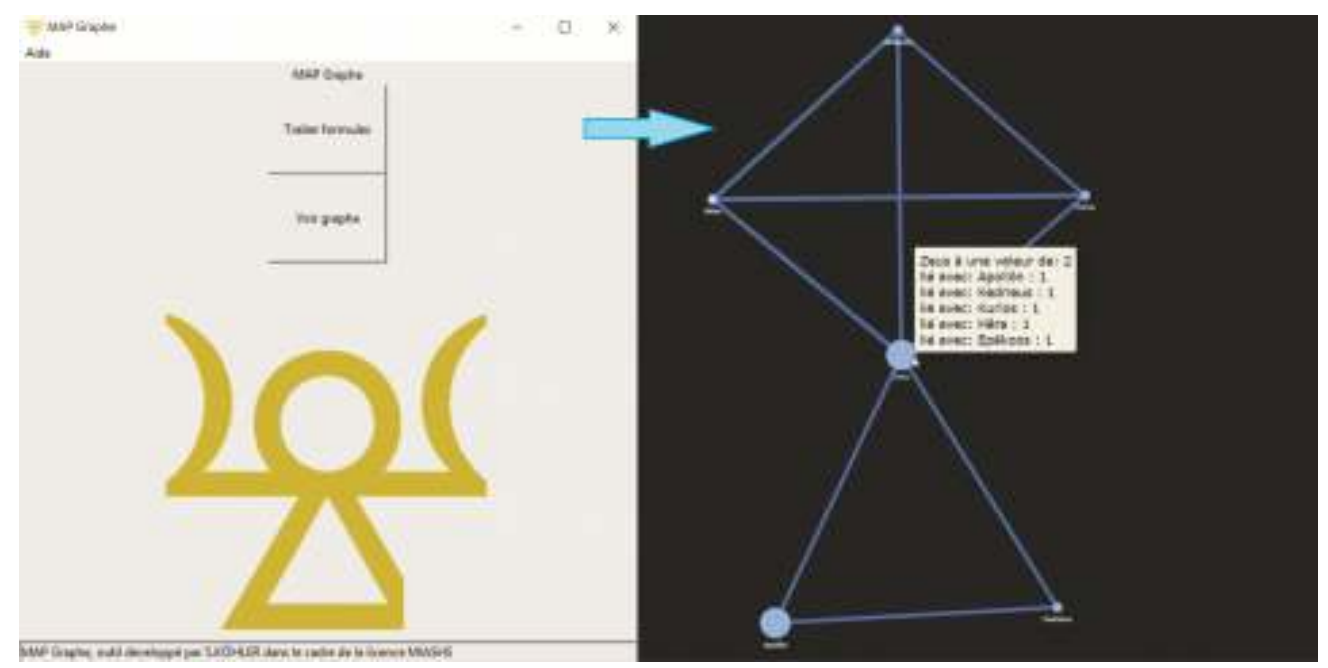

Projet ERC MAP. Licence Creative Commons BY-NC-SA 


\section{Conclusion}

Pour conclure, l'originalité du projet et des hypothèses qu'il sous-tend nous a amenés à tirer le meilleur parti des outils numériques. Le choix de ces derniers se fonde tant sur le FAIR data que sur les besoins propres du projet. Nécessaires à l'étude d'un corpus si vaste et hétéroclite, ces outils permettent également de questionner nos pratiques d'historien et notre rapport au numérique. En effet, avant de commencer le projet, l'ensemble de l'équipe était familier des recherches dans les bases de données en ligne, comme la BDEG ${ }^{14}$, la CGRN ${ }^{15}$ ou les IIP ${ }^{16}$. Cependant, ces bases ne portent pas spécifiquement sur les liens entre les dieux et l'espace et ne développent pas toujours d'interface cartographique. La création d'une base de données ad hoc et d'une interface de webmapping a ainsi nécessité de nombreuses heures de dialogue, de confrontation d'exemples, d'ontologies et de méthodologies dans chaque spécialité.

Actuellement, l'enregistrement des données occupe une grande partie de l'équipe, tandis que les outils d'analyse sont utilisés sur des corpus délimités temporellement ou spatialement. La prochaine étape est la mise en ligne en open access des données et des outils de requête et de $w e b$ mapping. Le projet MAP dispose également d'un programme de chercheurs invités, qui viennent à Toulouse travailler avec l'équipe pour enregistrer leur propre corpus au sein de la base de données. Ce dispositif permettra à terme d'assurer une saisie et une activité sur la base de données au-delà des cinq ans impartis au projet.

\section{Bibliographie}

Allen, Spencer L. 2015. The Splitered Divine. A Study of Istar, Baal and Yaweh Divine Names and Divine Multiplicity in the Ancient Near East. Berlin : De Gruyter.

Barré, Michael L. 1983. The God-List in the Treaty Between Hannibal and Philip V of Macedonia : A Study in Light of the Ancient Near Eastern Treaty Tradition. Baltimore : Johns Hopkins University Press.

Bonnet, Corinne. 2015. «Des chapelles d'or pour apaiser les dieux. Au sujet des aphidrymata carthaginois offerts à la métropole tyrienne en 310 av. J.-C. " Mythos. Rivista di Storia delle Religioni 9 : 71-86.

Bonnet, Corinne, Maria Bianco, Thomas Galoppin, Élodie Guillon, Antoine Laurent, Sylvain Lebreton et Fabio Porzia. 2018. "Les dénominations des dieux nous offrent comme autant d'images dessinées ». Studi e materiali di storia delle religioni 84 : 567-591.

Brunet, Roger, Robert Ferras et Hervé Théry, éd. 2005. Les Mots de la géographie. Dictionnaire critique. Montpellier et Paris : RECLUS et La Documentation Française.

Gómez Bellard, Carlos. 2008. « Ibiza. The Making of New Landscapes ». Dans Rural Landscapes of the Punic World, édité par Peter van Dommelen et Carlos Gómez Bellard, 44-75. Londres : Equinox.

Gómez Bellard, Carlos, Enrique Díes Cusí et Vicente Marí i Costa. 2011. Tres paisajes ibicencos : un estudio arqueológico. València : Universitat de València.

Guillon, Élodie. 2021. « Le monde clos des Pitiuses : essai d'analyse du fonctionnement territorial d'Ibiza et Formentera puniques ». Dans Insularidad, îléité y insularización en el Mediterráneo fenicio y púnico (actas del coloquio de los 29 y 30 de marzo 2017, Eivissa), édité par Benjamí Costa et Élodie Guillon, 183-200. Ibiza : Museo Arqueológico de Ibiza y Formentera.

Guillon, Élodie et Fabio Porzia. 2018. « Ancient Gods Going Digital. On the First Steps of the On-Going ERC Project "Mapping Ancient Polytheisms” ". Communication présentée à ASOR - EPHE-PSL European Symposium, Paris, 4-6 septembre. 
Köhler, Stanislas. 2019. « Développement de l'application Map Graphe ». Rapport de stage, université Toulouse-Jean-Jaurès.

Laurent, Antoine, Léa Vanessa, Vincent Hard, François Baleux, Carine Calastrenc, Ignacio Clemente Conte, Daniel Condis, Muriel Gandelin, Ermengol Gassiot Ballbè, Catherine Georjon, Florent Hautefeuille, Mélanie Le Couédic, Vivien Mathé, Clément Moreau, Joan Nunes Alonso, Frédéric Pouget et Christine Rendu. 2019. "Création de référentiels communs : la coordination complexe du travail collaboratif ». Archéologies numériques 3. https: //doi.org/10.21494/ISTE.OP.2019.0360.

Marín Ceballos, María Cruz, Ana María Jiménez Flores, María Belén Deamos, Jorge H. Fernández, Frédéric Horn et Ana Mezquida. 2010. "Les terres cuites de la grotte d'Es Culleram (Ibiza, Espagne) : iconographie et fonction ". Dans Figurines grecques en contexte. Présence muette dans le sanctuaire, la tombe et la maison, édité par Stéphanie HuysecomHaxhi et Arthur Muller, 133-157. Lille : Presses universitaires du Septentrion.

McCarter, Kyle. 1987. " Aspects of the Religion of the Israelite Monarchy ». Dans Ancient Israelite Religion : Essays in Honor of Frank Moore Cross, édité par Patrick D. Miller, Paul D. Hanson et Samuel Dean McBride, 125-136. Philadelphia : Fortress.

Parker, Robert. 2017. Greek Gods Abroad. Names, Natures and Transformations. Oakland : University of California Press.

Smith, Mark. 2016. Where the Gods Are. Spatial Dimensions of Anthropomorphism in the Biblical World. New Haven : Yale University Press.

Vernant, Jean-Pierre. 1965. Mythe et pensée chez les Grecs. Études de psychologie historique. Paris : François Maspéro.

Vernant, Jean-Pierre. 1974. « La société des dieux ». Dans Mythe et société en Grèce ancienne, édité par Jean-Pierre Vernant, 103-120. Paris : La Découverte.

\section{Notes}

1 Ce projet est financé par le Conseil européen de la Recherche (European Research Council [ERC]) dans le cadre du programme de recherche et d'innovation de l'Union européenne, Horizon 2020 (contrat de financement $\mathrm{n}^{\mathrm{o}} 741182$ ). Le projet est porté par Corinne Bonnet, professeure d'histoire grecque et spécialiste de l'histoire des religions du monde grec et du Proche-Orient, et comprend une équipe composée de quatre post-doctorants Aleksandra Kubiak-Schneider, spécialiste d'épigraphie araméenne et particulièrement de la Palmyrène, Fabio Porzia, historien spécialiste du Levant (pour les mondes sémitiques), Thomas Galoppin, spécialiste des pratiques « magiques » du monde romain et Sylvain Lebreton, spécialiste du polythéisme attique et de Zeus (pour les mondes grecs) -, d'une doctorante, Giuseppina Marano, et des auteurs. https://map-polytheisms.huma-num.fr.

2 L'auteur présente, à la suite de M.L. Barré (1983), K. McCarter (1987) et S.P. Allen (2015), une grammaire des expressions sémitiques associant un nom de dieu et un nom de lieu (voir p. 71-77).

3 PPI. Phéniciens et Puniques à Ibiza. Étude et modélisation spatiale du patrimoine archéologique d'Ibiza (VII ${ }^{e}-I^{e}$ siècle avant J.-C.). Projet de l'initiative d'excellence UNITI et financé au titre des actions thématiques stratégiques 2014, Toulouse, porté par C. Bonnet et É. Guillon. Voir https://ppi.hypotheses.org.

4 Précédemment, plusieurs spécialistes, dont C. Gómez Bellard (2008), avaient fait une hypothèse similaire, à partir de données archéologiques régionales.

5 L'équivalence est d'ailleurs faite dans l'Antiquité avec Tyché la grecque et Fortuna la romaine.

6 https://ineumann.developpez.com/tutoriels/merise/initiation-merise/.

7 https://pactols.frantiq.fr/opentheso/.

8 Huma-Num, l'infrastructure des humanités numériques : https://www.huma-num.fr.

9 Code source des interfaces du projet: https://github.com/LaboratoirePLH.

10 Looping-MCD, logiciel gratuit et libre d'utilisation (université Toulouse III) : http://ww w.looping-mcd.fr.

$11 \mathrm{https://www.postgresql.org.}$

12 https://symfony.com. Les interfaces ont été réalisées par Romain Gonçalves (https://rg oncalves.fr). 
13 https:/www.business-geografic.com/fr/ressources/documentation.html\#geo-technologies-business-geografic-geo-generateur. L’interface a été entièrement développée par l'auteur, A. Laurent.

14 BDEG : Banque de données des épiclèses grecques, développée par une équipe de l'université de Rennes dont faisait partie Sylvain Lebreton, actuellement post-doctorant travaillant au projet MAP : https://epiclesesgrecques.univ-rennes1.fr.

15 CGRN : Collection of Greek Ritual Norms, développée à l'université de Liège : http://cgr n.ulg.ac.be.

16 IIP : Inscriptions of Israel/Palestine : https://library.brown.edu/iip/index/. La base propose une interface de recherche cartographique.

\section{Auteurs}

\section{Élodie Guillon}

ERC MAP (741182), EA 4601 PLH - ERASME, université Toulouse-Jean-Jaurès, Toulouse, France

Élodie Guillon, spécialiste du Proche-Orient phénicien et de l'expansion phénicienne, est coordinatrice du projet ERC MAP, dans lequel elle est plus particulièrement en charge du développement du volet " humanités numériques ». Elle a précédemment mené plusieurs études comprenant l'application d'outils numériques (base de données, SIG, logiciels de statistiques multivariées) à des données archéologiques et historiques.

ORCID 0000-0001-8212-176X

elodie.guillon@univ-tlse2.fr

\section{Antoine Laurent}

ERC MAP (741182), EA 4601 PLH - ERASME, université Toulouse-Jean-Jaurès, Toulouse, France

Antoine Laurent est ingénieur de recherche, spécialiste du traitement de la donnée spatiale en archéologie et en histoire. Dans le projet ERC MAP, il est en charge du développement des outils numériques (base de données, webmapping, SIG). Il participe également à plusieurs autres projets au sein de l'université comme l'ANR Monumen, le GDR Chasseolab et l'étude $3 \mathrm{D}$ de la grotte Chauvet.

ORCID 0000-0002-3727-5644

antoine.laurent@univ-tlse2.fr

\section{Droits d'auteur}

Les contenus de la revue Humanités numériques sont mis à disposition selon les termes de la Licence Creative Commons Attribution 4.0 International. 